\title{
CHOP mediates ASPP2-induced autophagic apoptosis in hepatoma cells by releasing Beclin-1 from Bcl-2 and inducing nuclear translocation of $\mathrm{Bcl}-2$
}

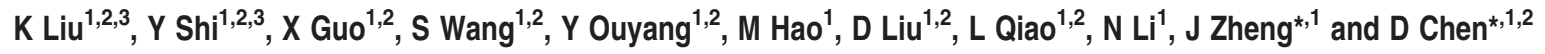

Apoptosis-stimulating protein of p53-2 (ASPP2) induces apoptosis by promoting the expression of pro-apoptotic genes via binding to p53 or p73; however, the exact mechanisms by which ASPP2 induces apoptotic death in hepatoma cells are still unclear. Here, we show that the transient overexpression of ASPP2 induces autophagic apoptosis in hepatoma cells by promoting p53- or p73-independent C/EBP homologous protein (CHOP) expression. CHOP expression decreases the expression of $\mathrm{Bcl}-2$; this change releases Beclin-1 from cytoplasmic Bcl-2-Beclin-1 complexes and allows it to initiate autophagy. However, transient overexpression of Beclin-1 can induce autophagy but not apoptosis. Our results show that ASPP2 induces the expression of damage-regulated autophagy modulator (DRAM), another critical factor that cooperates with free Beclin-1 to induce autophagic apoptosis. The effect of CHOP on the translocation and sequestration of $\mathrm{Bcl}-2$ in the nucleus, which requires the binding of Bcl-2 to ASPP2, is also critical for ASPP2-induced autophagic apoptosis. Although the role of nuclear ASPP2-Bcl-2 complexes is still unclear, our results suggest that nuclear ASPP2 can prevent the translocation of the remaining Bcl-2 to the cytoplasm by binding to Bcl-2 in a CHOP-dependent manner, and this effect also contributes to Beclin-1-initiated autophagy. Thus, CHOP is critical for mediating ASPP2-induced autophagic apoptosis by decreasing Bcl-2 expression and maintaining nuclear ASPP2-Bcl-2 complexes. Our results, which define a mechanism whereby ASPP2 overexpression induces autophagic apoptosis, open a new avenue for promoting autophagy in treatments to cure hepatocellular carcinoma.

Cell Death and Disease (2014) 5, e1323; doi:10.1038/cddis.2014.276; published online 17 July 2014

Apoptosis-stimulating protein of p53-2 (ASPP2) belongs to the evolutionarily conserved ASPP family. Members of this family are characterized by the presence of ankyrin repeats, an SH3 domain, and a protein-rich region. ${ }^{1,2}$ ASPP2 was previously identified as an interacting partner for several proteins, for example, Bcl-2 and p53. ${ }^{3}$ ASPP2 binds to p53 through its $C$ terminus to stimulate the transactivation function of p53 on the promoters of pro-apoptotic genes, such as the genes encoding Bax, PIG3 and PUMA. ASPP2 is a haploinsufficient tumor suppressor; ASPP2 ${ }^{+/-}$mice are prone to developing cancer. ${ }^{4}$ Recently, ASPP2 has been reported to bind RAS and to regulate autophagy by binding Atg5 via its $\mathrm{N}$ terminus. ${ }^{5,6}$

Autophagy is an evolutionarily conserved pathway for maintaining cellular homeostasis through the targeting of proteins and organelles to lysosomes for degradation. ${ }^{7}$ Upon induction, a small vesicular sac elongates and subsequently encloses a portion of the cytoplasm, forming a doublemembrane structure known as an autophagosome. The autophagosome then fuses with a lysosome to form an autolysosome in which the enclosed materials are degraded. ${ }^{8}$ The release of Beclin-1 from Bcl-2-Beclin-1 complexes is critical for inducing autophagy as free Beclin-1 is needed to interact with VPS34 and initiate autophagosome formation. ${ }^{9}$
Therapeutic approaches for hepatocellular carcinoma (HCC) that aim to increase the amount of autophagy have been successfully tested in vitro and in vivo. ${ }^{10}$ Furthermore, mice with heterozygous disruption of Beclin-1 have a high frequency of spontaneous HCC. ${ }^{11}$ These data suggest that maintaining and increasing autophagy is beneficial for preventing and curing $\mathrm{HCC}$.

The transcription factor C/EBP homologous protein (CHOP) regulates certain aspects of the cellular response to stress. ${ }^{12,13} \mathrm{CHOP}$ has been implicated in the regulation of energy metabolism, cellular proliferation and differentiation and the expression of cell type-specific genes. ${ }^{14} \mathrm{CHOP}$ expression is low in non-stressed conditions but is markedly increased in response to ER stress, amino-acid starvation and adipocytic differentiation. ${ }^{15-17}$ The overexpression of CHOP promotes apoptosis in several cell lines. ${ }^{18} \mathrm{CHOP}$ reduces the expression of $\mathrm{Bcl}-2$, an antiapoptotic factor that prevents the translocation of Bax to the mitochondria, which results in the induction of apoptosis. ${ }^{16} \mathrm{Bcl}-2$ is known to interact with Beclin-1, an initiator of autophagy. ${ }^{9}$ Autophagy is also regarded as an inducer of apoptosis, and a previous study reported that damage-regulated autophagy modulator (DRAM) expression is critical for inducing autophagic apoptosis. $^{8}$

\footnotetext{
${ }^{1}$ Beijing You'an Hospital, Capital Medical University, Beijing 100069, China and ${ }^{2}$ Beijing Institute of Hepatology, Beijing 100069, China

*Corresponding authors: D Chen or J Zheng, Beijing Institute of Hepatology, Beijing You'an Hospital, Capital Medical University, No. 8 Xi Tou Tiao, You An Men Wai, Feng Tai, Beijing 100069, China. Tel: +86 10 83997392; Fax: +86 10 63057109; E-mail: dexi0963@yahoo.com (DC) or Tel: +86 10 83997328; Fax: +86 10 83997628; E-mail: zhengjiasheng6@163.com (JZ)

${ }^{3}$ These authors contributed equally to this work.

Abbreviations: ASPP2, Apoptosis-stimulating protein of p53-2; HCC, hepatocellular carcinoma; DRAM, damage-regulated autophagy modulator; LC3, microtubuleassociated protein light chain 3; CHOP, C/EBP homologous protein; Bcl-2, B-cell lymphoma 2; Beclin-1, Bcl-2-interacting protein 1

Received 02.10.13; revised 25.5.14; accepted 29.5.14; Edited by GM Fimia
} 
The relationship between autophagy and apoptosis is still unclear, and the detailed mechanisms of ASPP2 function in autophagy and apoptosis have not been fully elucidated. In this study, we observed that transient overexpression of ASPP2 induces CHOP-mediated autophagic apoptosis in hepatoma cells.

\section{Results}

ASPP2 overexpression induces CHOP in a p53- or p73-independent manner; however, CHOP is still involved in ASPP2 overexpression-induced apoptosis in hepatoma cells. HCC cell lines (Hep1-6, HepG2, Hep3B and Huh7) were transfected with a plasmid expressing ASPP2 for $24 \mathrm{~h}$. We used immunofluorescence to observe that ASPP2 overexpression induced the expression of CHOP (Figures 1a and b, Supplementary Figure 1a). ASPP2 overexpression-induced CHOP was located mostly in the nucleus (Figure 1a). The vector did not induce CHOP expression in every hepatoma cell line (data not shown). The results of immunoblotting, M30 immunofluorescence and flow cytometry showed that ASPP2 overexpression promoted apoptosis in all cell lines (Figures 1c-e and Supplementary Figures $1 \mathrm{~b}$ and f). An MTT assay further showed that ASPP2 overexpression promoted cell death in all cell lines (Supplementary Figure 1c). Thus, ASPP2 overexpression induces apoptotic cell death in hepatoma cells. a
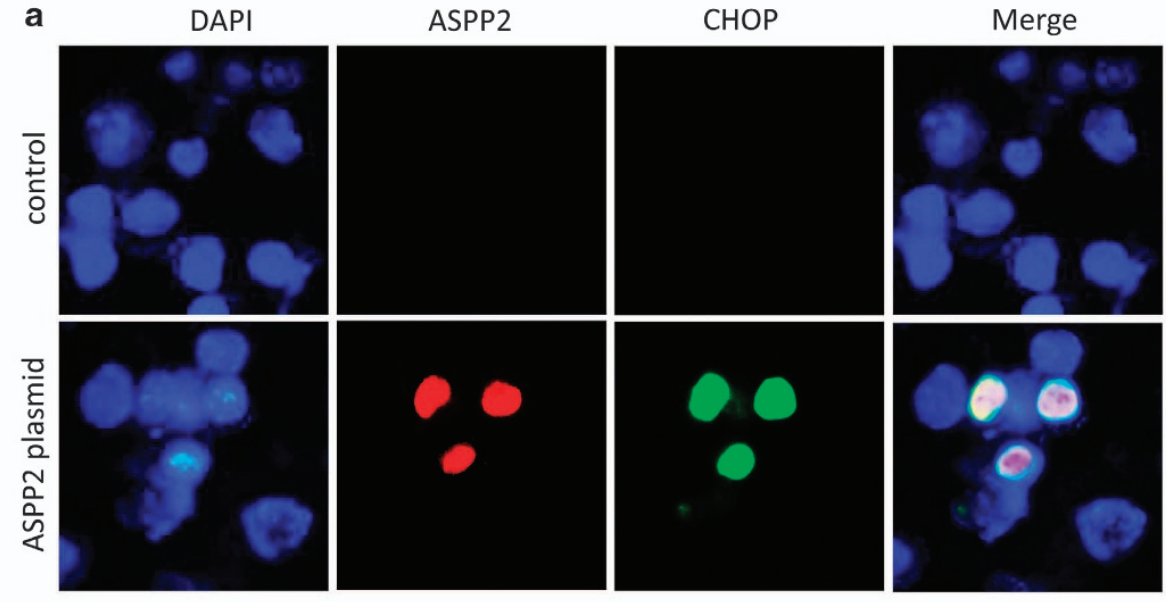
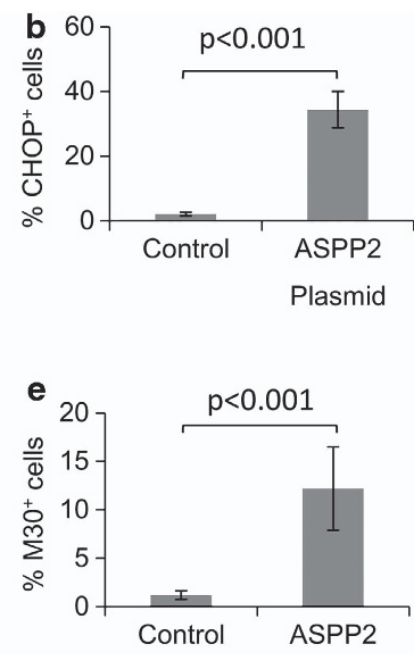

Plasmid
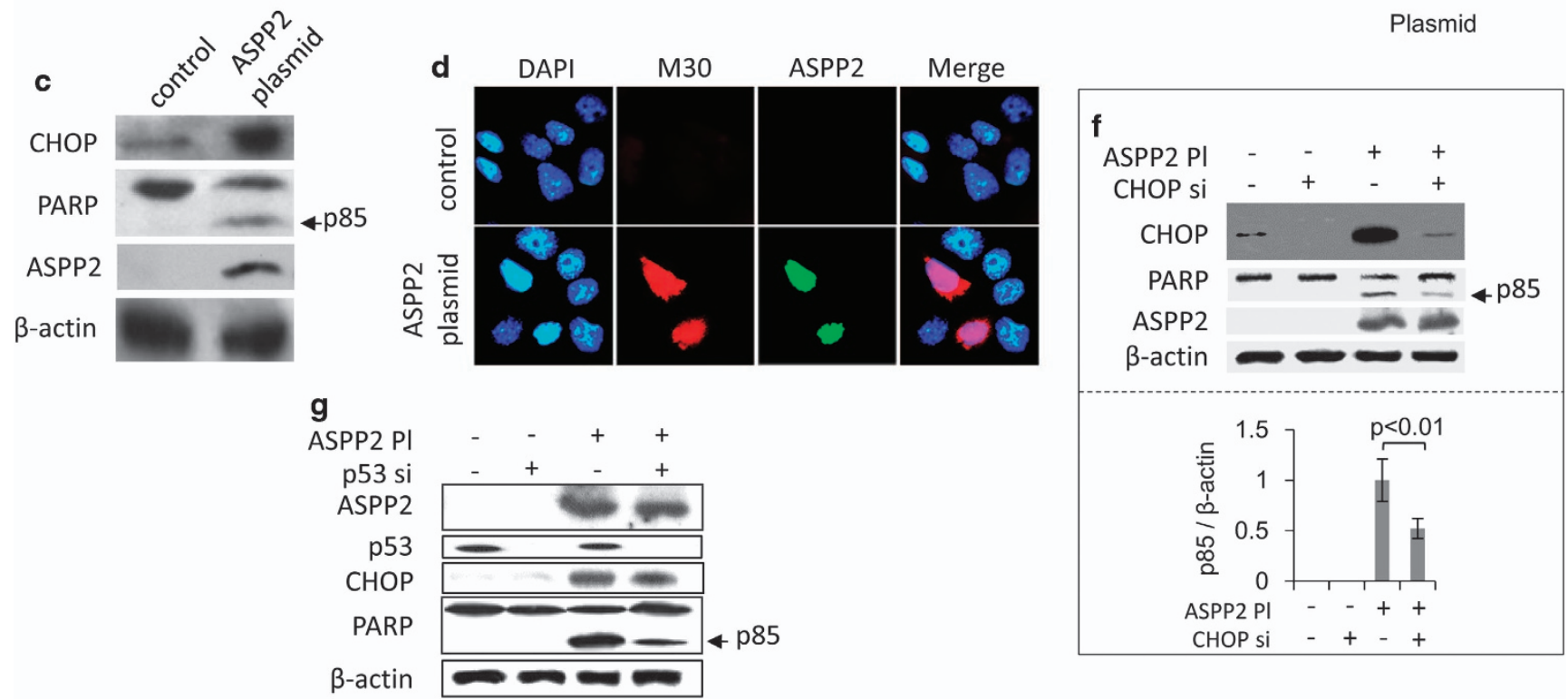

Figure 1 ASPP2 overexpression induces p53-independent CHOP-mediated apoptosis in Hep1-6 cells. Hep1-6 cells were transfected with a plasmid encoding ASPP2 (ASPP2 PI) for $24 \mathrm{~h}$. (a) Immunofluorescence detection of the expression of ASPP2 and CHOP. Nuclei were stained with 4',6-diamidino-2-phenylindole (DAPI). Magnification, $\times 400$. (b) Quantification of cells positive for CHOP expression as seen in a. Data are shown as the mean \pm S.E.M. of three independent experiments. (c) Immunoblot assay was used to evaluate the level of CHOP and cleaved PARP fragment (p85) in Hep1-6 cells using the indicated antibodies. (d) Immunofluorescence detection of M30-positive Hep1-6 cells. Nuclei were stained with DAPI. (e) Quantification of M30-positive cells as seen in $\mathbf{d}$. Data are shown as the mean \pm S.E.M. of three independent experiments. (f) Immunoblot assay was used to detect the effect of CHOP knockdown via siRNA (CHOP si) on apoptosis in Hep1-6 cells using the indicated antibodies (top panel). The ratio of $\mathrm{p} 85$ to $\beta$-actin was calculated from densitometry scanning data. Data are shown as the mean \pm S.E.M. of three independent experiments (bottom panel). (g) Immunoblot assay was used to detect the effect of p53 knockdown via siRNA (p53 si) on CHOP expression and apoptosis in Hep1-6 cells using the indicated antibodies 
The inhibition of CHOP expression via siRNA decreased the level of cleaved PARP fragment (p85) in Hep1-6, HepG2 and Hep3B cells, suggesting that ASPP2 overexpression can induce apoptosis in HCC by promoting CHOP expression (Figure 1f, Supplementary Figures 1d and f). We used p53 siRNA to inhibit p53 expression and observed that p53 knockdown failed to downregulate $\mathrm{CHOP}$ expression in Hep1-6 (Figure 1g) and HepG2 cells (Supplementary Figure 1e). We also failed to detect that p73 could mediate CHOP expression in Hep3B and Huh7 cells transfected with the ASPP2 plasmid (Figure $6 \mathrm{~b}$ and Supplementary Figure 1f). However, knockdown of p53 in Hep1-6 and HepG2 cells or knockdown of p73 in Hep3B and Huh7 cells decreased ASPP2 overexpression-induced apoptosis (Figures $1 \mathrm{~g}$ and 6b, Supplementary Figures 1e and f). Thus, although ASPP2 overexpression induces $\mathrm{CHOP}$ expression in a p53- or p73-independent manner, CHOP is still involved in ASPP2-induced p53- or p73-mediated apoptosis.

ASPP2-induced CHOP mediates autophagic apoptosis in hepatoma cells. $\mathrm{Bcl}-2$ is a classic antiapoptotic factor in cells. Previous studies have shown that $\mathrm{CHOP}$ can downregulate the level of $\mathrm{Bcl}-2$ expression. ${ }^{19}$ In this study, ASPP2 overexpression decreased the levels of $\mathrm{Bcl}-2$ mRNA and protein in Hep1-6 cells (Figures $2 \mathrm{a}$ and b). Bcl-2 is also reported to inhibit autophagy via binding to Beclin-1. Here, we failed to detect that ASPP2 overexpression significantly affected the levels of Beclin-1 mRNA and protein in Hep1-6 cells (Figures 2a and b). However, ASPP2 overexpression increased LC3 II expression, upregulated the ratio of LC3 II/I and decreased p62 level in Hep1-6 cells (Figures 2b and e and Supplementary Figure 2a). Exposure to BafA 1, an inhibitor of autophagic flux, increased the accumulation of LC3 II and p62 and upregulated the ratio of LC3 II/I in Hep1-6 cells with ASPP2 overexpression compared with cells that did not receive treatment with BafA 1 (Figure $2 \mathrm{c}$ and Supplementary Figure 2a). BafA 1 treatment had no effect on the expression of ASPP2 and CHOP (Figure 2c and Supplementary Figure 2c). We also detected that Atg5 knockdown via siRNA inhibited ASPP2-induced autophagy as shown by decrease of LC3 II and increase of p62 (Figure 2f). These data suggest that ASPP2 overexpression can induce autophagy in hepatoma cells.

Interestingly, using immunofluorescence, we observed that ASPP2 overexpression-induced GFP-LC3 II puncta were present in $\mathrm{HCC}$ cells positive for CHOP expression (Figure $3 \mathrm{~b}$ and Supplementary Figure $2 \mathrm{e}$ ). Knockdown of $\mathrm{CHOP}$ via siRNA significantly decreased the number of cells with GFPLC3 II puncta (Supplementary Figure 2e and f). Immunoblot assay also indicated that knockdown of CHOP via siRNA inhibited ASPP2 overexpression-induced autophagy in Hep1-6 cells (Figure 2d and Supplementary Figure 2b). Knockdown of CHOP via siRNA had no effect on the expression of ASPP2 and Beclin-1 in Hep1-6 cells with or without ASPP2 overexpression (Figure $2 d$ and Supplementary Figure 2d). Thus, CHOP is an ASPP2 overexpression-induced modulator of autophagy. As inhibition of autophagy via BafA 1 or Atg5 siRNA decreases ASPP2-induced apoptosis and cell death (Figures $2 f$ and $g$ and Supplementary Figure $2 g$ ), and CHOP knockdown via siRNA decreases ASPP2-induced autophagy and apoptosis, our data suggest that CHOP expression-induced autophagy is involved in ASPP2 overexpression-induced apoptosis. Furthermore, transfection with a plasmid encoding $\mathrm{Bcl}-2$ inhibited ASPP2 overexpressioninduced autophagy and apoptosis but had no effect on CHOP, Beclin-1 and ASPP2 levels (Figure 2e). We failed to detect that ASPP2 overexpression suppressed the AKT/mTOR axis (Supplementary Figure 4a). In addition, we also failed to detect that ASPP2 overexpression induced Tribbles homologue 3 (TRIB3) expression and that TRIB3 bound to AKT directly (Supplementary Figure 4b).

Taken together, our data suggest that ASPP2 overexpression induces CHOP expression, which mediates autophagic apoptosis in hepatoma cells. The mechanism by which $\mathrm{CHOP}$ induces autophagic apoptosis is associated with downregulation of $\mathrm{Bcl}-2$ expression.

ASPP2 overexpression releases Beclin-1 from Bcl-2-Beclin-1 complexes and the released Beclin-1 initiates ASPP2-induced autophagy. Using immunofluorescence, we determined that ASPP2 overexpression reduced $\mathrm{Bcl}-2$ expression and that, in the cytoplasm of Hep1-6 cells, localization of Bcl-2 and Beclin-1 did not overlap (Figure $3 \mathrm{a}$ ) as remaining $\mathrm{Bcl}-2$ translocated to the nucleus (Supplementary Figure 3a). Autophagy was induced in ASPP2- and CHOP-positive cells (Figure 3b). In Supplementary Figure 3b, we further identified that Beclin-1 colocalized with GFP-LC3 puncta in ASPP2-overexpressed cells, and Beclin-1 knockdown via siRNA reduced ASPP2induced GFP-LC3 puncta and GFP-LC3 puncta-positive cells (data not shown). Moreover, after transfection of Hep1-6 cells with a plasmid encoding $\mathrm{Bcl}-2$, we observed that $\mathrm{Bcl}-2$ overexpression rescued the colocalization of $\mathrm{Bcl}-2$ and Beclin-1 in the cytoplasm and inhibited autophagy (Supplementary Figures $3 \mathrm{~b}$ and $\mathrm{c}$ ). Immunoblot assays showed that Beclin-1 knockdown via siRNA reduced the levels of LC3 II and the ratio of LC3 II/I and increased the accumulation of p62 (Figures $3 c$ and d). These data suggest that the released Beclin-1 is involved in ASPP2 overexpression-induced autophagy. We also identified that knockdown of Beclin-1 via siRNA reduced ASPP2 overexpressioninduced cleavage of PARP ( $p 85$; Figures $3 c$ and e); however, Beclin-1 siRNA treatment itself had no effect on apoptosis (Figure 3f). Control siRNA treatment also had no effect on autophagy and apoptosis (Figure 3f). These data suggest that the downregulation of $\mathrm{Bcl}-2$ expression promotes the release of Beclin-1 from Bcl-2-Beclin-1 complexes; the released Beclin-1 then initiates ASPP2-induced autophagy, which induces apoptosis in hepatoma cells with ASPP2 overexpression.

CHOP is important for maintaining nuclear ASPP2-Bcl-2 complexes and for releasing Beclin-1 from Bcl-2-Beclin-1 complexes by downregulating $\mathrm{Bcl}-2$ expression. Previous results indicate that the remaining $\mathrm{Bcl}-2$ translocates to the nucleus in Hep1-6 cells after the overexpression of ASPP2. As the majority of ASPP2 also translocated to the nucleus when overexpressed, we investigated whether ASPP2 could bind $\mathrm{Bcl}-2$ in the extracted nuclei. Using an immunoprecipitation assay, we determined that ASPP2 overexpression 
increased the level of ASPP2-Bcl-2 complexes in the extracted nucleus of Hep1-6 cells, although $\mathrm{CHOP}$ expression dramatically decreased the overall level of $\mathrm{Bcl}-2$ (Figure 4a). Using an immunoblot assay to detect $\mathrm{Bcl}-2$ levels in the extracted nucleus and cytoplasm, we further identified that ASPP2 overexpression promoted the translocation of the remaining $\mathrm{Bcl}-2$ to the nucleus (Figure $4 \mathrm{~b}$ ). These data suggest that the majority of the transiently overexpressed ASPP2 binds Bcl-2 in the nucleus. A targeted siRNA knockdown of CHOP increased the total level of $\mathrm{Bcl}-2$ (Figure 2d); however, CHOP inhibition decreased the level of nuclear $\mathrm{Bcl}-2$ and increased the level of cytoplasmic Bcl-2 (Figure 4c). Moreover, immunoprecipitation results showed that the inhibition of $\mathrm{CHOP}$ expression decreased the level of nuclear ASPP2-Bcl-2 complexes and restored the level of cytoplasmic Bcl-2-Beclin-1 complexes (Figures 4d and e). Knockdown of CHOP did not affect the expression (Figure 2d and Supplementary Figure 2d) and distribution of ASPP2 (data not shown). Thus, CHOP is important for maintaining the interaction of ASPP2 and $\mathrm{Bcl}-2$ in the nucleus and for preventing the formation of $\mathrm{Bcl}-2-\mathrm{Beclin}-1$ complexes in the cytoplasm. Cytoplasmic Bcl-2 is an antiapoptotic factor, whereas nuclear $\mathrm{Bcl}-2$ can promote apoptosis. This suggests that the distinct distributions of $\mathrm{Bcl}-2$ have differing functions. ${ }^{20}$ Moreover, we did not detect that CHOP could directly bind to ASPP2, Bcl-2 or Beclin-1 (Supplementary Figure 5).
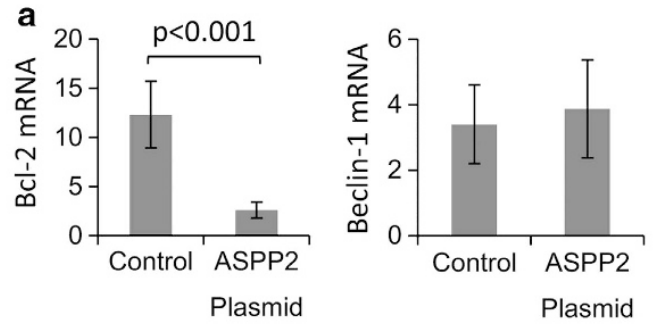
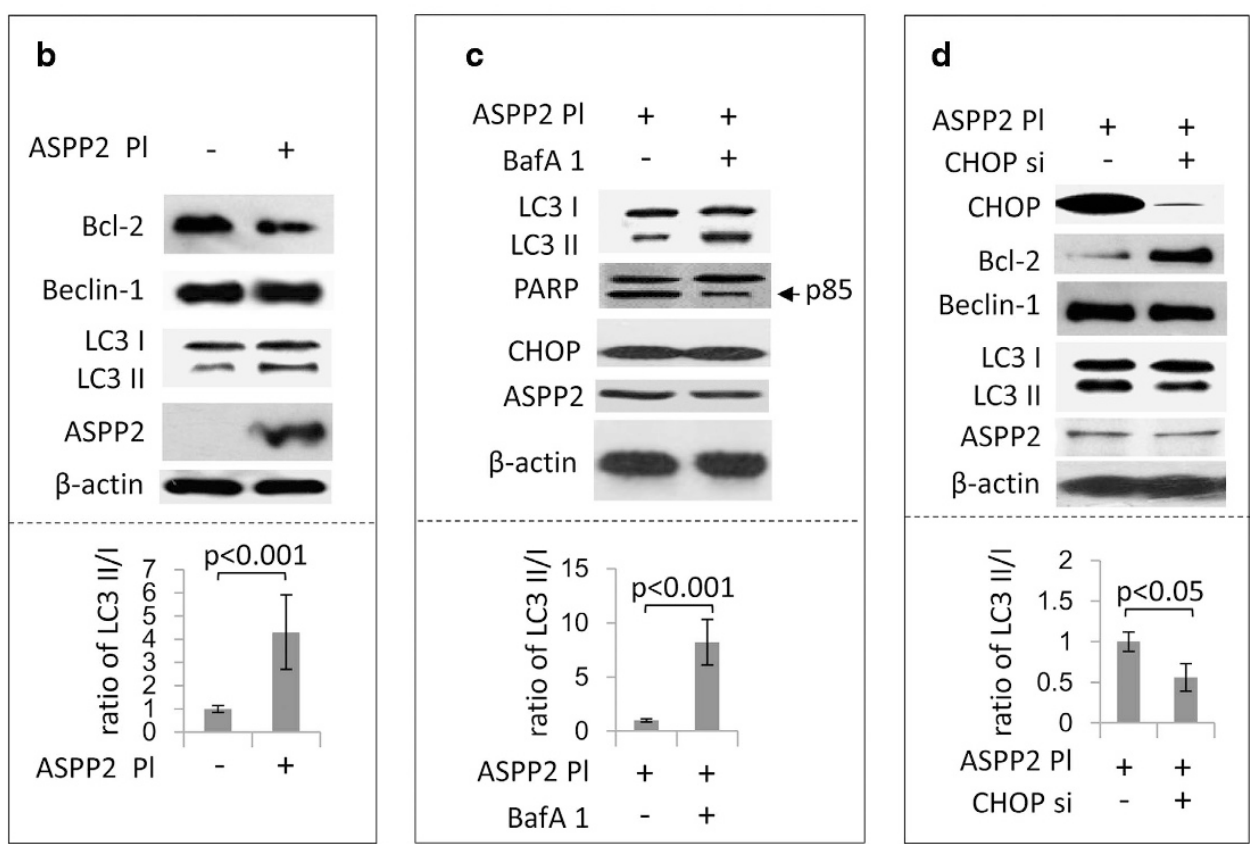

Figure 2 CHOP expression decreases BCL-2 expression and induces autophagic apoptosis. (a) Real-time PCR was used to detect Bcl-2 and Beclin-1 mRNA levels in Hep1-6 cells with or without transfection with a plasmid encoding ASPP2 (ASPP2 PI) for $24 \mathrm{~h}$. (b) Immunoblot showing the effects of ASPP2 overexpression on Bcl-2, Beclin-1 and LC3 I/II levels in Hep1-6 cells (top panel). The ratio of LC3 II/I was calculated from densitometry scanning data (bottom panel). (c) Immunoblot showing autophagy inhibition via Bafilomycin A1 (BafA 1) on the expression levels of PARP, CHOP and ASPP2 in Hep1-6 cells (top panel). The ratio of LC3 I//I was calculated from densitometry scanning data (bottom panel). (d) Immunoblot showing the effects of CHOP knockdown via siRNA (CHOP si) on Bcl-2, Beclin-1, LC3 I/II and ASPP2 levels in Hep1-6 cells (top panel). The ratio of LC3 II/I was calculated from densitometry scanning data (bottom panel). (b-d) Data of the ratio of LC3 II/I are shown as the mean \pm S.E.M. of three independent experiments. (e) Immunoblot assay was used to detect the effect of Bcl-2 overexpression on the levels of LC3 I/II, p62, ASPP2, CHOP and Beclin-1 in Hep1-6 cells with or without transfection of the ASPP2 plasmid. (f) Immunoblot assay was used to detect the effect of Atg5 knockdown via siRNA (Atg5 si) on the level of autophagy and apoptosis in Hep1-6 cells with or without transfection of ASPP2 PI. (g) MTT assay was used to detect the effect of BafA 1 (upper panel) or Atg5 si pre-treatment (lower panel) on cell viability in Hep1-6, HepG2, Hep3B and Huh7 cells with or without transfection of the ASPP2 plasmid 

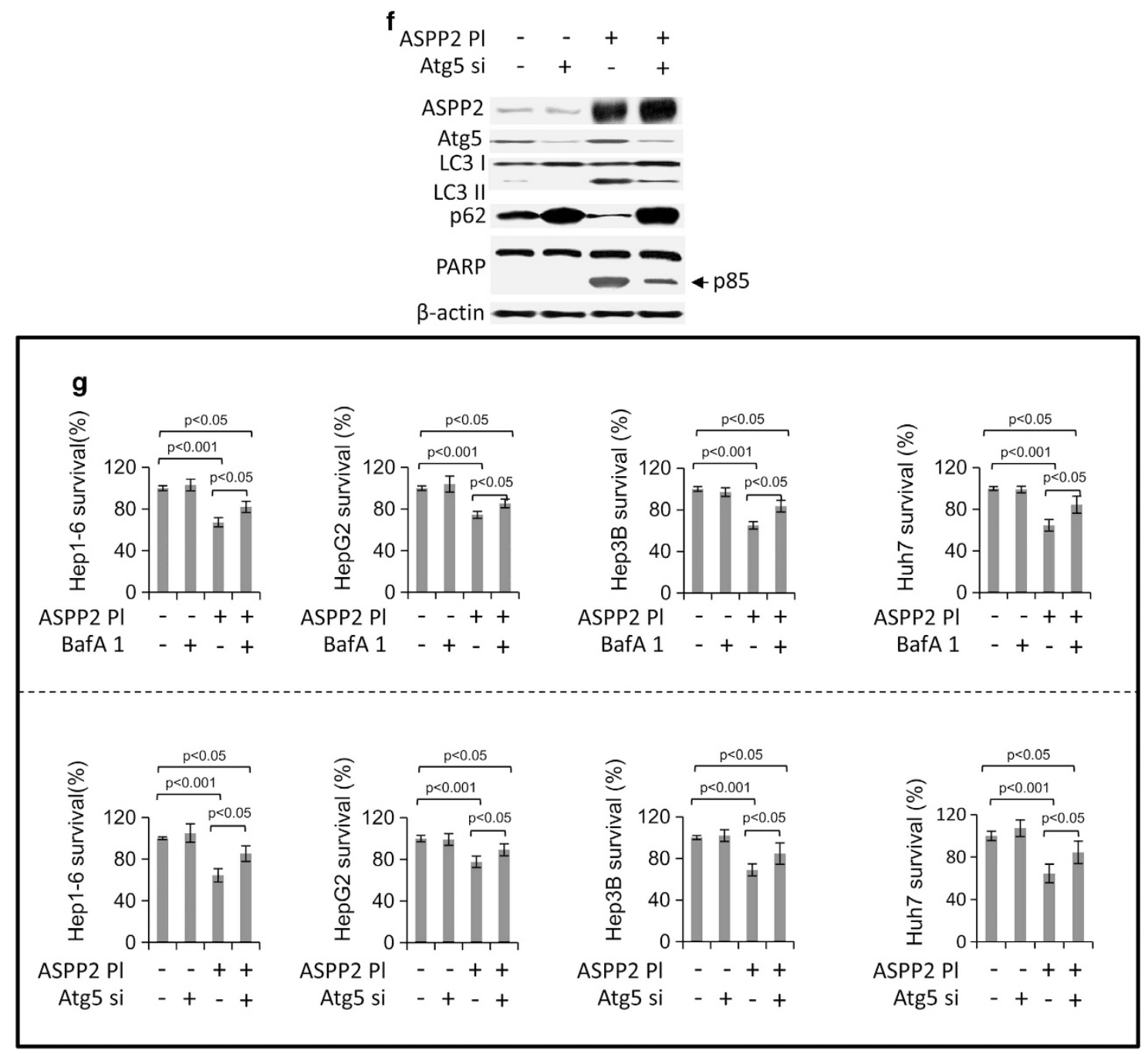

Figure 2 (Continued)

Thus, our results suggest that the transient overexpression of ASPP2 can suppress cytoplasmic Bcl-2 by binding to it in the nucleus in a CHOP-dependent manner.

Beclin-1-initiated autophagy is involved in ASPP2-induced apoptosis but is not sufficient for its induction. We have shown that Beclin-1 is released from cytoplasmic Bcl-2-Beclin-1 complexes and can then initiate ASPP2 overexpression-induced autophagy in HCC cells. Accordingly, the transient overexpression of Beclin-1 enhanced ASPP2 overexpression-induced apoptosis by promoting increased autophagy development in Huh7 and Hep1-6 cells in a CHOP-independent manner (Figures $5 \mathrm{a}, \mathrm{b}, \mathrm{d}$ and e). These results suggest that Beclin-1-initiated autophagy is necessary for ASPP2-induced apoptosis. However, when Huh7 and Hep1-6 cells were transfected with the Beclin-1 plasmid solely, Beclin-1 overexpression induced autophagy but not apoptosis, suggesting that promoting Beclin-1initiated autophagy is not sufficient for the induction of apoptosis (Figures $5 \mathrm{c}$ and $\mathrm{f}$ ). Moreover, as knockdown of p53 in Hep1-6 cells and knockdown of p73 in Hep3B cells decrease ASPP2 overexpression-induced autophagic apoptosis (Figure 1g, Supplementary Figures $1 \mathrm{f}$ and g), we believe that other factors might be involved in ASPP2induced autophagic apoptosis and these factors might be regulated by $\mathrm{p} 53$ or $\mathrm{p} 73$.

ASPP2 induces CHOP-independent expression of DRAM, and the ASPP2-induced DRAM promotes autophagic apoptosis. Previous studies have shown that DRAM expression induces autophagy and that DRAMinduced autophagy is an inducer of apoptosis. ${ }^{8,21}$ In this study, we observed that ASPP2 overexpression increased DRAM mRNA and protein in Hep1-6, Huh7 and Hep3B cells (Figures $6 a$ and $b$ and Supplementary Figure 1f). By using siRNA to knockdown DRAM expression, we detected decreased levels of ASPP2-induced apoptosis and autophagy, suggesting that DRAM is involved in ASPP2-induced autophagic apoptosis (Figures $6 \mathrm{c}$ and $\mathrm{d}$ and Supplementary Figure 1f). Moreover, we detected that ASPP2-induced DRAM expression was p53-dependent in Hep1-6 and HepG2 cells and was p73-dependent in Huh7 and Hep3B cells (Figure 6b, Supplementary Figures 1e-g). Immunoblot assay showed that DRAM knockdown via siRNA did not affect 

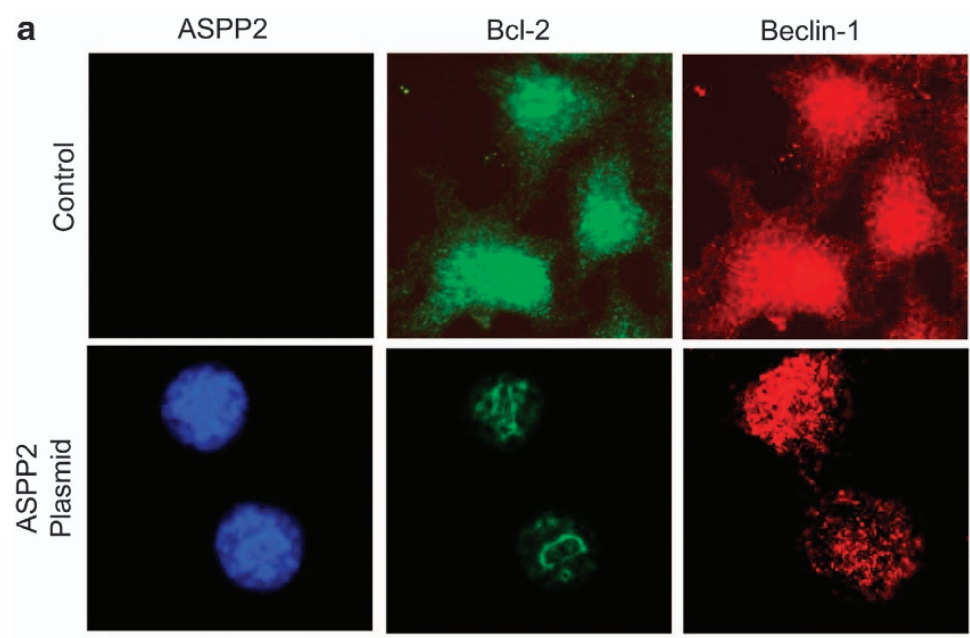

b
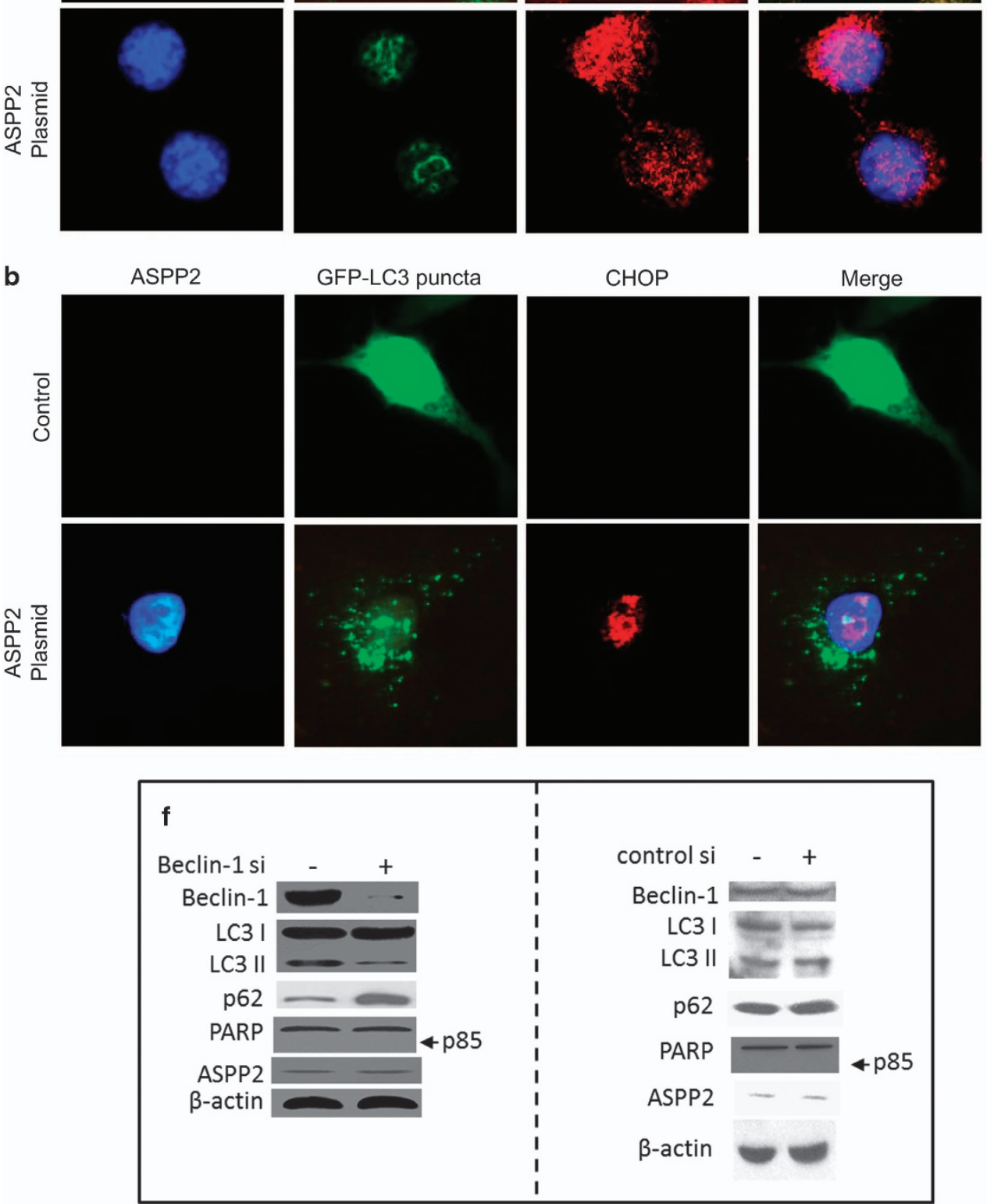

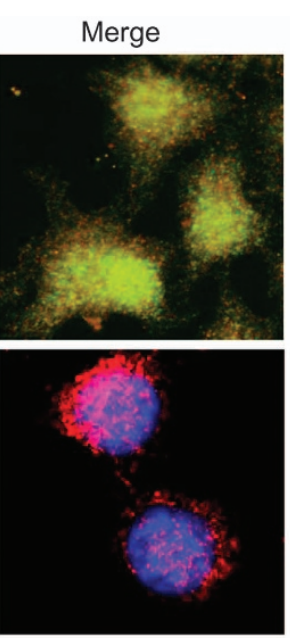

C
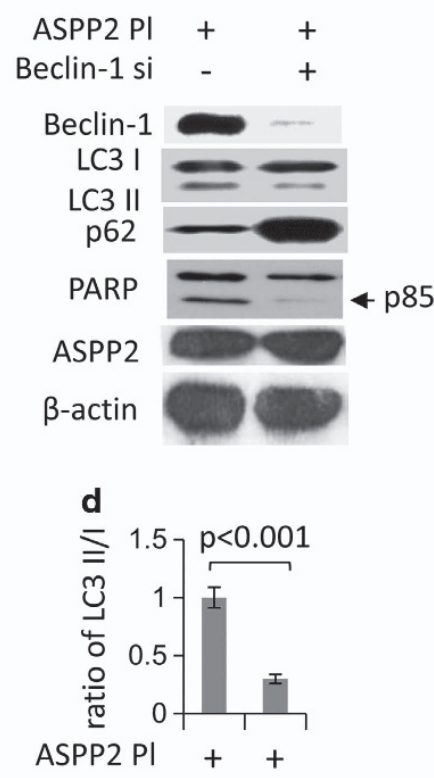

Beclin-1 si - +

e

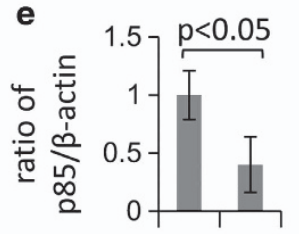

ASPP2 Pl ++

Beclin-1 si - +

Figure 3 ASPP2 overexpression releases Beclin-1 from cytoplasmic Bcl-2-Beclin-1 complexes and then the released Beclin-1 induces autophagy. Hep1-6 cells were transfected with a plasmid encoding ASPP2 for $24 \mathrm{~h}$. ( $\mathbf{a}$ and $\mathbf{b}$ ) Fluorescence microscopy was used to detect the colocalization of ASPP2 (blue), Bcl-2 (green) and Beclin-1 (red) (a) or the colocalization of ASPP2 (blue), GFP-LC3 puncta (green) and CHOP (red) (b). Magnification, $\times 1000$. (c) Hep1-6 cells were transfected with a plasmid encoding ASPP2 (ASPP2 PI) or co-transfected with the ASPP2 PI and Beclin-1 siRNA (Beclin-1 si) for $24 \mathrm{~h}$. Immunoblot assay was used to detect levels of LC3 l/II, p62, PARP and ASPP2. (d and e) The ratio of LC3 II/I (d) and p85/ $\beta$-actin (e) was calculated from densitometry scanning data in $\mathbf{c}$. Data of the ratio of $L C 3 \mathrm{ll} / \mathrm{l}$ and $p 85 / \beta$-actin are shown as the mean \pm S.E.M. of three independent experiments. (f) Immunoblot assay was used to detect the effects of Beclin-1 si (left panel) or control siRNA (control si, right panel) treatment on autophagy, apoptosis and ASPP2 expression in Hep1-6 cells

CHOP expression in Hep1-6, Huh7 and Hep3B cells with or without ASPP2 overexpression, suggesting that $\mathrm{CHOP}$ expression is independent of DRAM (Figure $6 \mathrm{c}$ and
Supplementary Figure 1f). The results of immunoblotting and immunofluorescence assays also showed that $\mathrm{CHOP}$ did not affect DRAM expression in Hep1-6 and Huh7 cells 

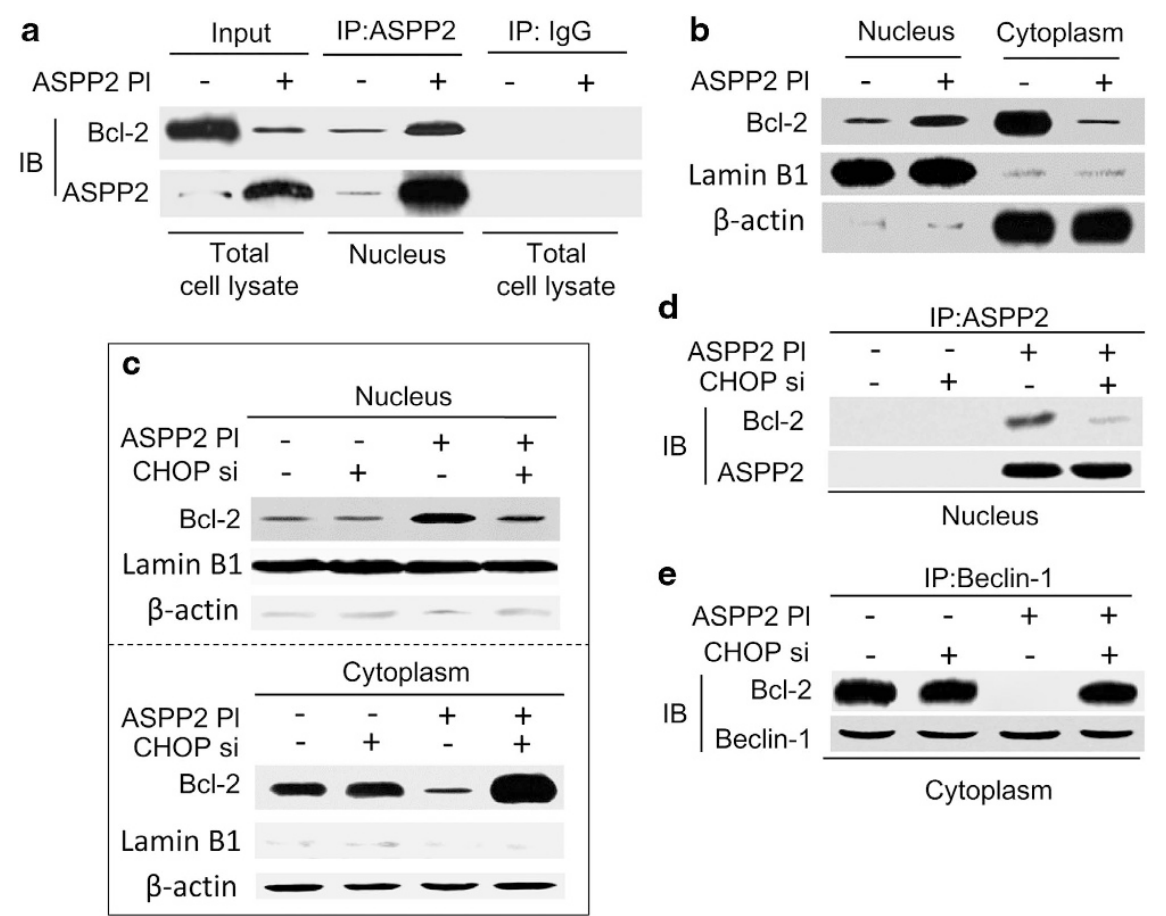

d

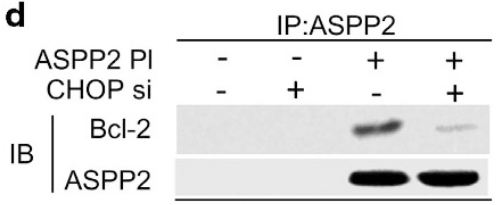

Nucleus

e

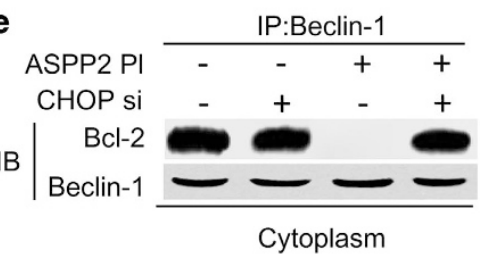

Figure 4 CHOP is critical for maintaining nuclear ASPP2-Bcl-2 complexes. (a) Hep1-6 cells were transfected with the ASPP2 plasmid (ASPP2 PI) for $24 \mathrm{~h}$ and then nuclei were extracted. Anti-ASPP2 antibody was used to immunoprecipitate ASPP2 in the extracted nuclei. Total cell lysates were used as input or were immunoprecipitated with control IgG as indicated. Bcl-2 and ASPP2 were then analyzed using immunoblot assay. (b and $\mathbf{c}$ ) Hep1-6 cells were transfected with the ASPP2 PI (b) or co-transfected with ASPP2 PI and CHOP siRNA (CHOP si) for $24 \mathrm{~h}$ (c). Nuclei and cytoplasm were then extracted. Immunoblot assay was used to detect Bcl-2 level in the extracted nuclei and cytoplasm. (b) Anti-lamin B1 and anti- $\beta$-actin antibodies were used as controls for the extracted nuclei and cytoplasm, respectively. (d and e) Hep1-6 cells were transfected with ASPP2 PI or co-transfected with ASPP2 Pl and CHOP si for $24 \mathrm{~h}$, and then the nuclei (d) and cytoplasm (e) were extracted. In the extracted nuclei, anti-ASPP2 antibody was used to immunoprecipitate ASPP2. Bcl-2 and ASPP2 were then analyzed using immunoblot assay (d). In the extracted cytoplasm, anti-Beclin-1 antibody was used to immunoprecipitate Beclin-1. Bcl-2 and Beclin-1 were then analyzed using immunoblot assay (e)

with or without transfection with the ASPP2 plasmid, implying that DRAM expression is independent of CHOP (Figures $7 \mathrm{a}-\mathrm{e}$ ). We also did not detect that DRAM could affect the interaction of $\mathrm{Bcl}-2$ with Beclin-1 or the interaction of ASPP2 with Bcl-2 (Supplementary Figures $6 \mathrm{a}$ and $\mathrm{b}$ ). Moreover, simultaneous knockdown of p53 and CHOP in Hep1-6 cells or simultaneous knockdown of p73 and CHOP in Hep3B cells could produce a stronger inhibition of autophagy and apoptosis than knockdown of p53/p73 or CHOP alone, suggesting that both CHOP and p53/p73 have a major role in inducing autophagic apoptosis in response to ASPP2 overexpression (Supplementary Figure 7). Taken together, we suggest that p53- or p73-mediated DRAM expression and CHOP-released Beclin-1 act in concert to induce autophagic apoptosis in hepatoma cells when ASPP2 is transiently overexpressed. In addition, CHOP promotes the interaction between ASPP2 and Bcl-2 in the nucleus, which also contributes to ASPP2-induced autophagic apoptosis (Figure 7f).

\section{Discussion}

ASPP2, an important inducer of apoptosis, has been reported to inhibit the growth of hepatoma cells in vitro and in vivo. ${ }^{22}$ Here, we show that ASPP2 can induce apoptosis via the induction of autophagy. Conversely, a recent study on the relationship between ASPP2 and autophagy concluded that ASPP2 inhibits autophagy by binding Atg5 via its $\mathrm{N}$ terminus. ${ }^{6}$
This discrepancy may be explained by a difference in the genetic backgrounds of the cell lines studied. The contradictory results highlight the complex relationship between ASPP2 expression and autophagy.

Our results show that ASPP2-mediated autophagy is dependent on CHOP expression. To our knowledge, few studies have focused on the relationship between ASPP2 and $\mathrm{CHOP}$. We show that transfecting hepatoma cell lines with an ASPP2 plasmid induces CHOP expression and nuclear localization regardless of the cellular p53 status, suggesting that $\mathrm{CHOP}$ expression occurs in a p53-independent manner. As a multifunctional transcription factor, $\mathrm{CHOP}$ mediates gene expression by complex mechanisms. As a member of the C/EBP family, CHOP binds to other members of the C/EBP family to form heterodimers that promote or inhibit gene expression. ${ }^{16,17,23} \mathrm{CHOP}$ can also modulate the activity of other bZIP transcription factors that do not belong to the C/EBP family. ${ }^{24}$ Many studies have demonstrated that $\mathrm{CHOP}$ promotes apoptosis by decreasing Bcl-2 expression, ${ }^{16}$ and our data also support this hypothesis.

As a classic antiapoptotic factor, $\mathrm{Bcl}-2$ prevents $\mathrm{Bax}$ oligomerization in the mitochondrial outer membrane. ${ }^{25}$ However, the translocation of $\mathrm{Bcl}-2$ to the nucleus is reported to induce apoptosis. ${ }^{20}$ Correspondingly, our results show that, although the level of $\mathrm{Bcl}-2$ significantly decreases following the expression of $\mathrm{CHOP}$, the remaining $\mathrm{Bcl}-2$ does translocate to the nucleus, where it binds to ASPP2. Moreover, the persistence of 

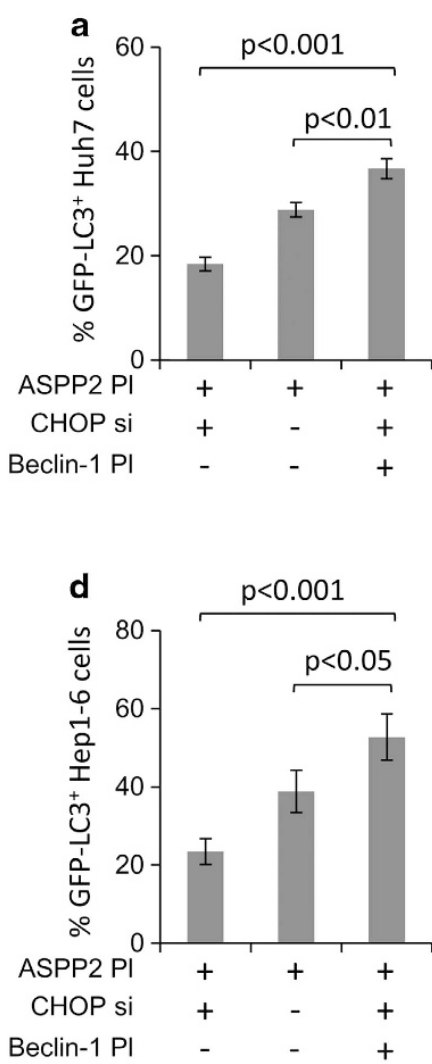

b

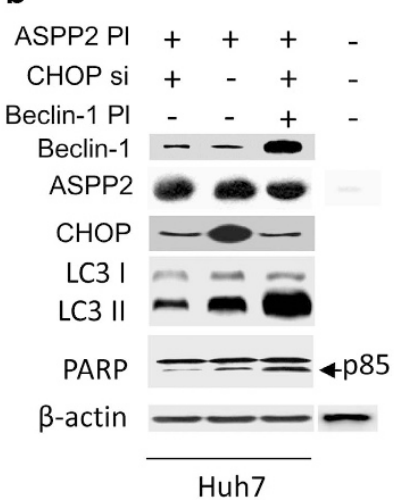

e



C

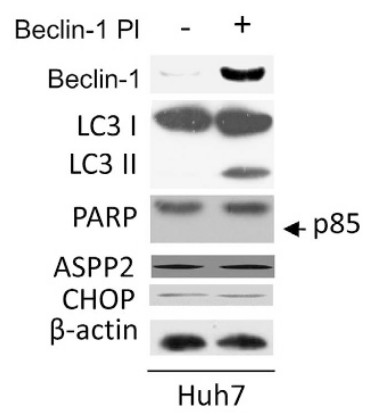

f

Beclin-1 PI - +

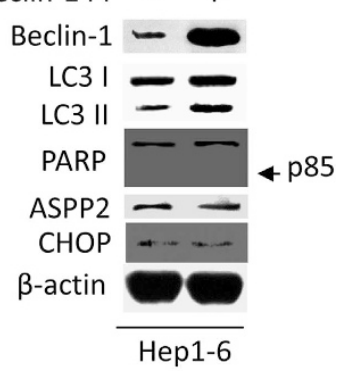

Figure 5 Beclin-1-initiated autophagy is involved in ASPP2-induced apoptosis but is not sufficient for its induction. (a, b, d and e) Huh7 (a and b) and Hep1-6 cells (d and $\mathbf{e}$ ) were transfected with a plasmid encoding GFP-LC3 or co-transfected with one or more of the indicated plasmids or siRNA (including the ASPP2 plasmid (ASPP2 PI), the Beclin-1 plasmid (Beclin-1 PI) and CHOP siRNA (CHOP si)). Immunofluorescence assay was used to evaluate the rate of GFP-LC3 puncta-positive Huh7 (a) and Hep1-6 cells (d). Quantification of Huh7 (a) and Hep1-6 cells (d) with more than 10 GFP-LC3 puncta. Data are shown as the mean \pm S.E.M. of three independent experiments. (b and $\mathbf{e}$ ) Immunoblot assay was used to detect autophagy and apoptosis using the indicated antibodies in Huh7 (b) and Hep1-6 cells (e). (c and f) Immunoblot detection of Beclin-1 overexpression on autophagy and apoptosis in Huh7 (c) and Hep1-6 cells (f) using the indicated antibodies

CHOP is critical for maintaining nuclear-localized Bcl-2. Although we do not know the role of nuclear ASPP2-Bcl-2 complexes, retaining Bcl-2 in the nucleus by binding to ASPP2 prevents the cytoplasmic translocation of $\mathrm{Bcl}-2$, where it functions as a well-known antiapoptotic factor. ${ }^{20}$

The translocation of $\mathrm{Bcl}-2$ to the nucleus also downregulates the level of cytoplasmic Bcl-2-Beclin-1 complexes. Beclin-1 is an initiator of autophagy and an inhibitor of tumorigenesis; it is thought to be a haploinsufficient tumor suppressor. ${ }^{26}$ Our results show that ASPP2 overexpression does not affect Beclin-1 expression levels but does result in the release of Beclin-1 from cytoplasmic Bcl-2-Beclin-1 complexes and allows released Beclin-1 to initiate ASPP2induced autophagy. Previous studies of Beclin-1 $1^{+/-}$mutant mice have demonstrated that Beclin-1 is a tumor-suppressor gene. ${ }^{11,27}$ Further studies of mice with depletion of Atg5 or Atg7 also found that autophagy deficiency induces liver tumors. ${ }^{28}$ In our study, we determined that transient overexpression of Beclin-1 initiates autophagy but that this autophagy does not promote apoptotic cell death. We also demonstrated that Beclin-1-initiated autophagy participates in ASPP2-induced apoptosis regardless of CHOP status. These data show that the relationship between Beclin-1-induced autophagy and apoptosis is complicated and suggest that the induction of apoptosis involves additional factors.
The exact mechanisms by which autophagy induces apoptosis or protects cells from stress are still unclear. To our knowledge, DRAM is one of the first genes reported to be associated with autophagic apoptosis. Inducing DRAM expression promotes autophagic apoptosis in a p53-dependent manner. ${ }^{8}$ In a previous study, we demonstrated that DRAM-mediated autophagy is involved in fatty acid-induced apoptosis in hepatoma cells. ${ }^{21}$ However, the exact mechanisms of apoptosis induction by DRAM-mediated autophagy are still unclear. In this study, we showed that the transfection of hepatoma cells with an ASPP2 plasmid promotes DRAM expression and that the resulting DRAMmediated autophagy promotes apoptosis. Another study demonstrated that DRAM expression can be induced by $p 73$ when p53 is deficient but that p73-dependent DRAM expression does not induce autophagic apoptosis. ${ }^{29}$ Our results show that ASPP2 overexpression induces DRAM expression by activating p53 in HepG2 and Hep1-6 cells or by activating p73 in Hep3B and Huh7 cells, which is then able to induce autophagic apoptosis. Although the exact mechanisms of autophagy-induced apoptosis in hepatoma cells require further study, our results uncover a new relationship between ASPP2 and autophagic apoptosis. ASPP2-induced autophagic apoptosis via the promotion of CHOP expression should be investigated further as it is a novel, targetable, signaling 

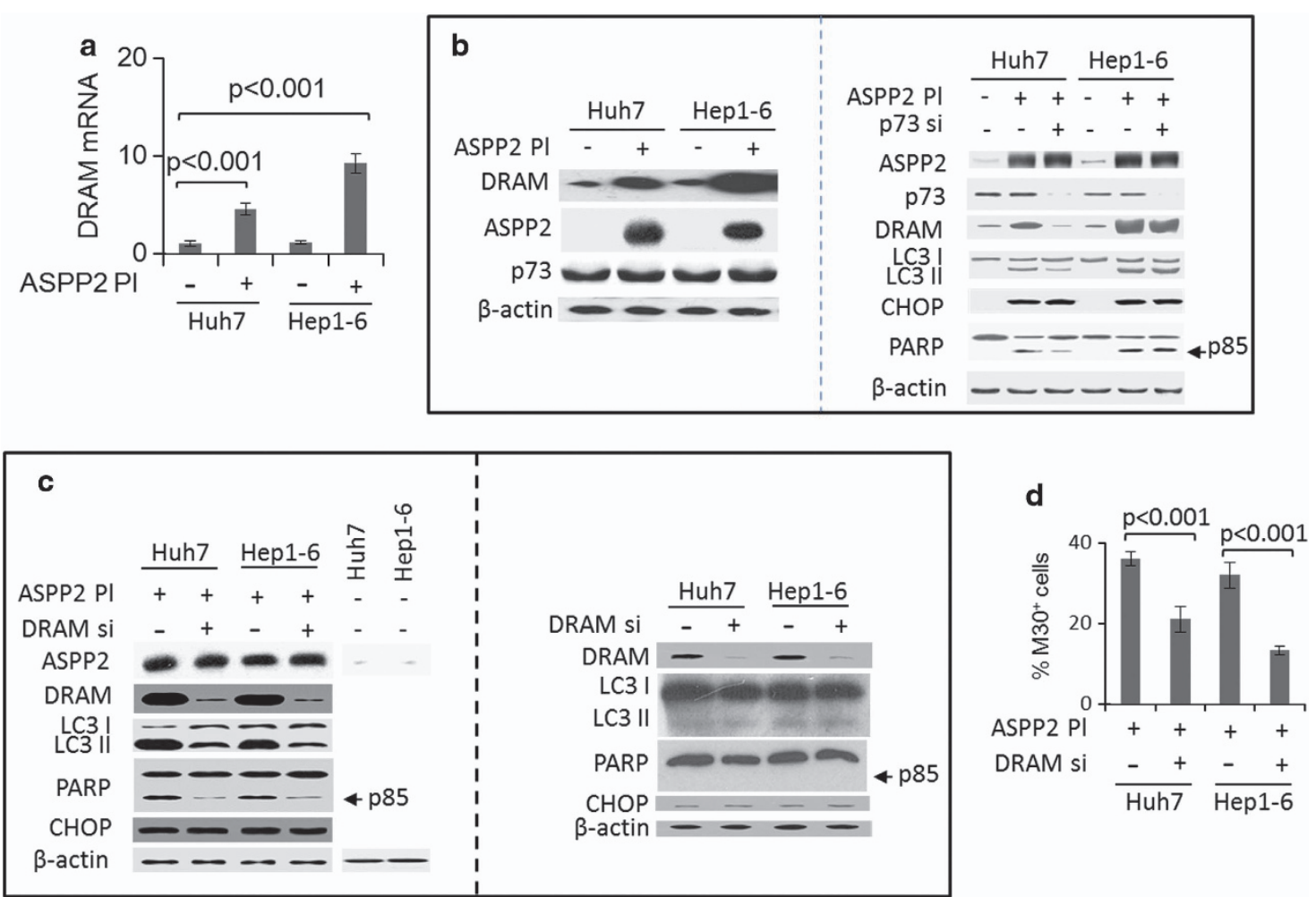

Figure 6 ASPP2 overexpression induces DRAM-mediated autophagic apoptosis. (a and b) Hep1-6 and Huh7 cells were transfected with the ASPP2 plasmid (ASPP2 PI) for $24 \mathrm{~h}$ with or without co-transfection with p73 siRNA (p73 si). Real-time PCR and immunoblot assays were used to detect the mRNA (a) and protein (b, left panel) level of DRAM in Huh7 and Hep1-6 cells. Immunoblot assay was used to detect the effect of p73 si on DRAM, LC3 I/II, CHOP and PARP levels in Huh7 and Hep1-6 cells (b, right panel). (c) Immunoblot assay was used to detect the effects of DRAM knockdown via siRNA (DRAM si) on CHOP expression and the levels of autophagy and apoptosis in Hep1-6 and Huh7 cells with (left panel) or without (right panel) transfection with ASPP2 PI. (d) M30 assay was used to detect the effects of DRAM knockdown on autophagy and apoptosis. (a and $\mathbf{d})$ Data are shown as the mean \pm S.E.M. of three independent experiments

step that could be useful in the improvement of therapies for HCC.

\section{Materials and Methods}

Cell culture and treatment. Hep1-6, HepG2 and Huh7 cells were grown in Dulbecco's modified Eagle's medium (DMEM), and Hep3B cells were grown in modified Eagle's medium (MEM). DMEM and MEM were supplemented with $10 \%$ fetal bovine serum. All cells were seeded in 6- or 24-well plates, transfected for $24 \mathrm{~h}$ with plasmids expressing ASPP2, Beclin-1, Bcl-2 and GFP-LC3 with Fugene HD (Promega, Madison, WI, USA) or with p53, CHOP, Beclin-1, Atg5 and DRAM siRNAs using Lipofectamine 2000 (Invitrogen, Carlsbad, CA, USA). The sequences of DRAM siRNA were derived from Crighton et al. ${ }^{8}$ The siRNAs of p53, CHOP, Atg5 and Beclin-1 were purchased from Santa Cruz Biotechnology Inc. (Santa Cruz, CA, USA). Bafilomycin A1 (BafA 1) was used to inhibit autophagic flux. Cells were grown on glass cover slips for immunofluorescence studies.

Immunoblot assay. Cell lysates, extracted nuclei and cytoplasm were subjected to western blot analysis, as described previously. ${ }^{21}$ Briefly, proteins was separated on $10-15 \%$ SDS-PAGE gels, and the separated proteins were then transferred to PVDF membranes. The protein blots were blocked with $5 \%$ milk and probed sequentially with specific primary antibodies and horseradish peroxidaseconjugated secondary antibodies. The detection of specific proteins on the blots was achieved with enhanced chemiluminescence (Pierce SuperSignal, Thermo Fisher Scientific Inc., Rockford, IL, USA), and the results were captured on X-ray films. Densitometric analysis was performed using the Image-Pro Plus analysis software (Media Cybernetics, Inc., Rockville, MD, USA).

Real-time PCR. The RNeasy Mini Kit (Qiagen, Hilden, Germany) was used to isolate total RNA from cultured cells. Reverse transcription to synthesize first-strand cDNA was conducted using the SuperScript II First-stand Synthesis System for RT-PCR (Invitrogen). SYBR Green was used to detect the dsDNA products during the real-time PCR reaction. The mRNA content was normalized to the housekeeping gene $\beta$-actin. ${ }^{21}$ The specific primer sequences used for real-time PCR were as follows: for BCl-2, $5^{\prime}$-GAGTACCTGAACCGGCACCT-3' (forward) and 5'-TCCCAGCCTCCGTTATCCT-3' (reverse); for Beclin-1, 5'-CTGG GACAACAAGTTTGACCAT- $3^{\prime}$ (forward) and $5^{\prime}$-GCTCCTCAGAGTTAAACTG GGTT-3' (reverse); for DRAM, 5'-TCAAATATCACCATTGATTTCTGT-3' (forward) and $5^{\prime}$-GCCACATACGGATGGTCATCTCTG- $3^{\prime}$ (reverse) (the sequences of the DRAM primers are per Crighton et al. $\left.{ }^{8}\right)$; and for $\beta$-actin, $5^{\prime}$-GCCCTGAGGC ACTCTTCCA-3' (forward) and 5'-CGGATGTCCACGTCACACTT-3' (reverse).

Fluorescence microscopy. Frozen cells were fixed with 10\% paraformaldehyde/PBS, incubated in $1 \%$ Triton X-100/PBS for 5 min, blocked with $3 \%$ BSA/PBS and probed with antibodies against ASPP2, CHOP, Bcl-2 and Beclin-1. Cy3, FITC, Dylight 405-conjugated secondary antibodies were used to amplify the signal. Nuclei were counterstained with 4',6-diamidino-2-phenylindole. The M30 mouse antibody was produced by our laboratory and was used to detect cleaved keratin 18 in early apoptotic cells. Fluorescence microscopy was used to detect the colocation of Bcl-2 and Beclin-1 or the colocation of GFP-LC3 puncta and Beclin-1 or the colocation of ASPP2 and Bcl-2. For quantitative apoptosis analysis, at least 500 cells per sample were counted.

Subcellular fractionation. The cytoplasm and nuclei were extracted using a Percoll gradient centrifugation adapted from previous methods. ${ }^{30}$ Briefly, cells were Dounce homogenized on ice in M-SHE buffer $(0.21 \mathrm{~mol} / / \mathrm{mannitol}, 0.07 \mathrm{~mol} / \mathrm{l}$ sucrose, $10 \mathrm{mmol} / / \mathrm{HEPES}-\mathrm{KOH}(\mathrm{pH} 7.4), 1 \mathrm{mmol} / / \mathrm{EDTA}, 1 \mathrm{mmol} / \mathrm{I}$ EGTA, $0.15 \mathrm{mmol} / /$ spermine, $0.75 \mathrm{mmo} / /$ spermidine and $1 \mathrm{mmol} / / \mathrm{DTT}$ ) with freshly added protease inhibitors $(1 \mu \mathrm{g} / \mathrm{ml}$ of leupeptin, aprotinin, pepstatin $\mathrm{A}, 1 \mathrm{mmol} / \mathrm{l}$ phenylmethylsulfonyl fluoride). Nuclei were pelleted at $1200 \times g$, and the supernatant was taken as the cytoplasmic fraction. 
a

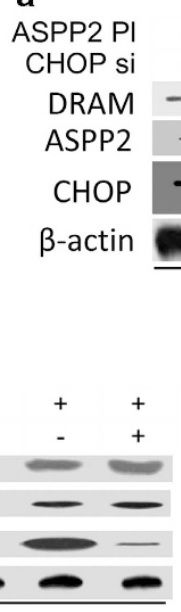

Huh7

C

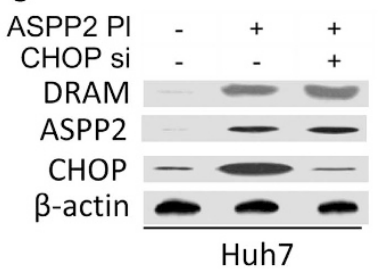

d

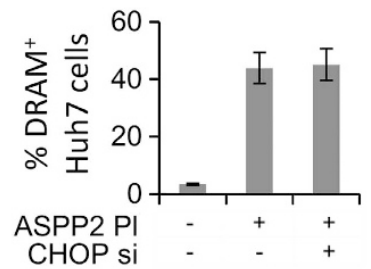

b

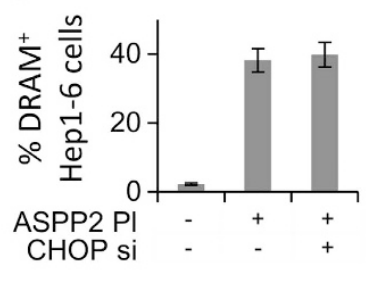

f

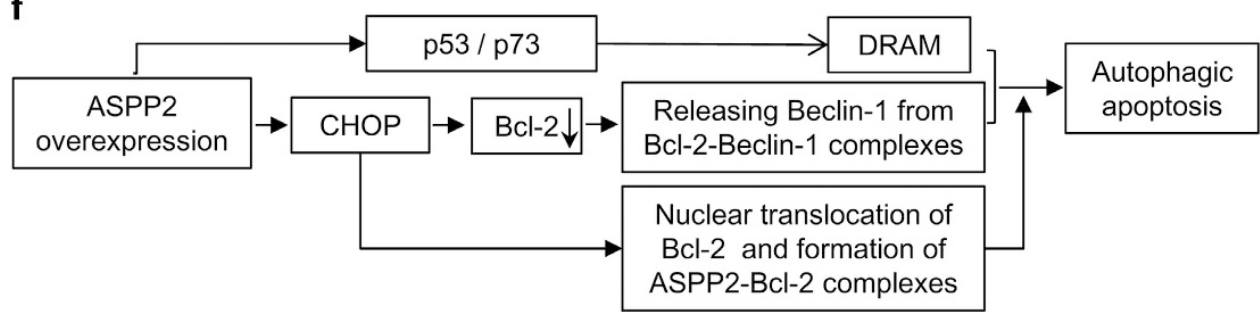

Figure 7 CHOP does not affect the expression of DRAM. (a-d) Hep1-6 and Huh7 cells were transfected with the ASPP2 plasmid (ASPP2 PI) with or without co-transfection with CHOP siRNA (CHOP si). (a and c) Immunoblot assay was used to detect the levels of DRAM, CHOP and ASPP2 in Hep1-6 (a) and Huh7 cells (c). (b and d) Immunofluorescence assay was used to detect cells positive for DRAM expression in Hep1-6 (b) and Huh7 cells (d). Data are shown as the mean \pm S.E.M. of three independent experiments. (e) Hep1-6 and Huh7 cells were transfected with CHOP si, and then immunoblot was used to detect the expression of DRAM and CHOP. (f) Diagram summarizes our data (details are provided in the Results section)

Immunoprecipitation assay. Cell lysates, extracted nuclei and cytoplasm ( $500 \mu \mathrm{g}$ of protein in $500 \mu \mathrm{l}$ of RIPA lysis buffer) were pre-cleared using protein A/G PLUS-agarose beads (Santa Cruz Biotechnology Inc.) and were incubated at $4{ }^{\circ} \mathrm{C}$ overnight with anti-ASPP2 or anti-Beclin-1 antibodies. Immunocomplexes were separated by incubation with protein $A / G$ agarose beads and were resolved using SDS-PAGE. Immunoblot assay was performed using the anti-Bcl-2 antibody.

Cell viability and apoptosis analysis. Cell viability was quantified with the MTT colorimetric assay kit (Promega), and cell apoptosis was detected by flow cytometric analysis following staining with Annexin V/PI (BD PharMingen, San Diego, CA, USA), according to the instructions of the manufacturer.

Statistical analysis. All data shown are the results of at least three independent experiments and are expressed as the mean \pm S.E.M. The differences between groups were compared using Student's $t$-test. Differences were considered significant at confidence levels of $P<0.05, P<0.01$ and $P<0.001$, as indicated.

\section{Conflict of Interest}

The authors declare no conflict of interest.

Acknowledgements. This work was supported in part by the National Natural Science Foundation of China (81272266, 81361120401, 81100288 and 81071843) and by The National Key Technology R\&D Program (2012BAl15B09).

1. Bergamaschi D, Samuels $Y$, O'Neil NJ, Trigiante G, Crook T, Hsieh JK et al. iASPP oncoprotein is a key inhibitor of p53 conserved from worm to human. Nat Genet 2003; 33 : 162-167.
2. Samuels-Lev Y, O'Connor DJ, Bergamaschi D, Trigiante G, Hsieh JK, Zhong S et al. ASPP proteins specifically stimulate the apoptotic function of p53. Mol Cell 2001; 8: 781-794.

3. Bergamaschi D, Samuels Y, Jin B, Duraisingham S, Crook T, Lu X. ASPP1 and ASPP2: common activators of p53 family members. Mol Cell Biol 2004; 24: 1341-1350.

4. Kampa KM, Acoba JD, Chen D, Gay J, Lee H, Beemer K et al. Apoptosis-stimulating protein of p53 (ASPP2) heterozygous mice are tumor-prone and have attenuated cellular damage-response thresholds. Proc Natl Acad Sci USA 2009; 106: 4390-4395.

5. Wang Z, Liu Y, Takahashi M, Van Hook K, Kampa-Schittenhelm KM, Sheppard BC et al. $\mathrm{N}$ terminus of ASPP2 binds to Ras and enhances Ras/Raf/MEK/ERK activation to promote oncogene-induced senescence. Proc Natl Acad Sci USA 2013; 110: 312-317.

6. Wang Y, Wang XD, Lapi E, Sullivan A, Jia W, He YW et al. Autophagic activity dictates the cellular response to oncogenic RAS. Proc Natl Acad Sci USA 2012; 109: $13325-13330$.

7. Klionsky DJ, Abdalla FC, Abeliovich H, Abraham RT, Acevedo-Arozena A, Adeli K et al. Guidelines for the use and interpretation of assays for monitoring autophagy. Autophagy 2012; 8: 445-544

8. Crighton D, Wilkinson S, O'Prey J, Syed N, Smith P, Harrison PR et al. DRAM, a p53-induced modulator of autophagy, is critical for apoptosis. Cell 2006; 126: 121-134.

9. Marquez RT, Xu L. Bcl-2:Beclin 1 complex: multiple, mechanisms regulating autophagy/ apoptosis toggle switch. Am J Cancer Res 2012; 2: 214-221.

10. Rautou PE, Mansouri A, Lebrec D, Durand F, Valla D, Moreau R. Autophagy in liver diseases. J Hepatol 2010; 53: 1123-1134.

11. Qu X, Yu J, Bhagat G, Furuya N, Hibshoosh H, Troxel A et al. Promotion of tumorigenesis by heterozygous disruption of the beclin 1 autophagy gene. J Clin Invest 2003; 112: 1809-1820.

12. Zinszner H, Kuroda M, Wang X, Batchvarova N, Lightfoot RT, Remotti $\mathrm{H}$ et al. CHOP is implicated in programmed cell death in response to impaired function of the endoplasmic reticulum. Genes Dev 1998; 12: 982-995.

13. Tabas I, Ron D. Integrating the mechanisms of apoptosis induced by endoplasmic reticulum stress. Nat Cell Biol 2011; 13: 184-190.

14. Tsukada J, Yoshida Y, Kominato Y, Auron PE. The CCAAT/enhancer (C/EBP) family of basic-leucine zipper (bZIP) transcription factors is a multifaceted highly-regulated system for gene regulation. Cytokine 2011; 54: 6-19. 
15. Harding HP, Zhang $Y$, Bertolotti A, Zeng $H$, Ron D. Perk is essential for translational regulation and cell survival during the unfolded protein response. Mol Cell 2000; 5: 897-904.

16. Ron D, Habener JF. CHOP, a novel developmentally regulated nuclear protein that dimerizes with transcription factors C/EBP and LAP and functions as a dominant-negative inhibitor of gene transcription. Genes Dev 1992; 6: 439-453.

17. Wang XZ, Kuroda M, Sok J, Batchvarova N, Kimmel R, Chung P et al. Identification of novel stress-induced genes downstream of chop. EMBO J 1998; 17: 3619-3630.

18. Kim I, Xu W, Reed JC. Cell death and endoplasmic reticulum stress: disease relevance and therapeutic opportunities. Nat Rev Drug Discov 2008; 7: 1013-1030.

19. Oyadomari S, Mori M. Roles of CHOP/GADD153 in endoplasmic reticulum stress. Cell Death Differ 2004; 11: 381-389.

20. Portier BP, Taglialatela G. Bcl-2 localized at the nuclear compartment induces apoptosis after transient overexpression. J Biol Chem 2006; 281: 40493-40502.

21. Liu K, Lou J, Wen T, Yin J, Xu B, Ding W et al. Depending on the stage of hepatosteatosis, p53 causes apoptosis primarily through either DRAM-induced autophagy or BAX. Liver Int 2013; 33: 1566-1574.

22. Zhao J, Wu G, Bu F, Lu B, Liang A, Cao L et al. Epigenetic silence of ankyrin-repeatcontaining, SH3-domain-containing, and proline-rich-region- containing protein 1 (ASPP1) and ASPP2 genes promotes tumor growth in hepatitis B virus-positive hepatocellular carcinoma. Hepatology 2010; 51: 142-153.

23. Ubeda M, Wang XZ, Zinszner H, Wu I, Habener JF, Ron D. Stress-induced binding of the transcriptional factor CHOP to a novel DNA control element. Mol Cell Biol 1996; 16 : 1479-1489.

24. Ubeda M, Vallejo M, Habener JF. CHOP enhancement of gene transcription by interactions with Jun/Fos AP-1 complex proteins. Mol Cell Biol 1999; 19: 7589-7599.

25. Mikhailov V, Mikhailova M, Pulkrabek DJ, Dong Z, Venkatachalam MA, Saikumar P. Bcl-2 prevents Bax oligomerization in the mitochondrial outer membrane. J Biol Chem 2001; 276 18361-18374.
26. Liang $\mathrm{XH}$, Jackson $\mathrm{S}$, Seaman $\mathrm{M}$, Brown $\mathrm{K}$, Kempkes $\mathrm{B}$, Hibshoosh $\mathrm{H}$ et al. Induction of autophagy and inhibition of tumorigenesis by beclin 1. Nature 1999; 402: 672-676.

27. Yue Z, Jin S, Yang C, Levine AJ, Heintz N. Beclin 1, an autophagy gene essential for early embryonic development, is a haploinsufficient tumor suppressor. Proc Natl Acad Sci USA 2003; 100: 15077-15082.

28. Takamura A, Komatsu M, Hara T, Sakamoto A, Kishi C, Waguri S et al. Autophagydeficient mice develop multiple liver tumors. Genes Dev 2011; 25: 795-800.

29. Crighton D, O'Prey J, Bell HS, Ryan KM. p73 regulates DRAM-independent autophagy that does not contribute to programmed cell death. Cell Death Differ 2007; 14: 1071-1079.

30. Chen D, Yu Z, Zhu Z, Lopez CD. The p53 pathway promotes efficient mitochondrial DNA base excision repair in colorectal cancer cells. Cancer Res 2006; 66: 3485-3494.

(c) (i) $(\Theta)$ Cell Death and Disease is an open-access journal published by Nature Publishing Group. This work is licensed under a Creative Commons Attribution-NonCommercialNoDerivs 3.0 Unported License. The images or other third party material in this article are included in the article's Creative Commons license, unless indicated otherwise in the credit line; if the material is not included under the Creative Commons license, users will need to obtain permission from the license holder to reproduce the material. To view a copy of this license, visit http://creativecommons.org/ licenses/by-nc-nd/3.0/

Supplementary Information accompanies this paper on Cell Death and Disease website (http://www.nature.com/cddis) 